\title{
Commissioning of CMS Forward Hadron Calorimeters with Upgraded Multi-anode PMTs and $\mu$ TCA Readout
}

\author{
Emrah Tiras $^{* 1}$, Burak Bilki ${ }^{1,2}$, Yasar Onel ${ }^{1}$ \\ ${ }^{1}$ University of Iowa, Iowa City, IA, USA \\ ${ }^{2}$ Beykent University, Istanbul, Turkey
}

On behalf of the CMS Collaboration

University of Iowa, Iowa City, IA, USA

E-mail: emrah-tiras@uiowa.edu

\begin{abstract}
The high flux of charged particles interacting with the CMS Forward Hadron Calorimeter PMT windows introduced a significant background for the trigger and offline data analysis. During Long Shutdown 1, all of the original PMTs were replaced with multi-anode, thin window photomultiplier tubes. At the same time, the back-end electronic readout system was upgraded to $\mu \mathrm{TCA}$ readout. The experience with commissioning and calibration of the Forward Hadron Calorimeter is described as well as the $\mu \mathrm{TCA}$ system. The upgrade was successful and provided quality data for Run 2 data-analysis at $13 \mathrm{TeV}$.
\end{abstract}

38th International Conference on High Energy Physics 3-10 August 2016

Chicago, USA

\footnotetext{
* Speaker.
} 


\section{Introduction}

The increasing luminosity and resulting radiation conditions entail upgrades of detectors at the Large Hadron Collider (LHC). The upgrade of the Compact Muon Solenoid (CMS) detector includes upgrading many sub-detectors such as Forward Hadron (HF) Calorimeter in the eta region, $3<\eta<5$ [1]. The HF calorimeter is made of steel absorbers and quartz fibers as active elements to generate photons as particles traverse. These photons are collected with 1728 photomultiplier tubes (PMTs).

The previous single-anode Hamamatsu R7525 PMTs were decided to be replace with new multi-anode, metal package Hamamatsu R7600U-200-M4 PMTs because of the large energy events detected by the former PMTs [1,2]. The source of the large energy events is high energy muons produced in pp collisions, cosmic particles, and charged particles from late showering hadrons; which produce $\breve{C}$ erenkov light at the window of the PMTs. Figure 1 shows the muon response of HF R7525 PMTs at $150 \mathrm{GeV}$ muon test beam during 2004-2008. In the plot, $200 \mathrm{GeV}$ peak is due to muons interacting with the PMT windows and the sharp peak around $7 \mathrm{GeV}$ is due to muons interacting with the HF calorimeter itself. This confirms that the glass window of the previous R7525 PMTs are susceptible to the muons and they needed to be replaced.

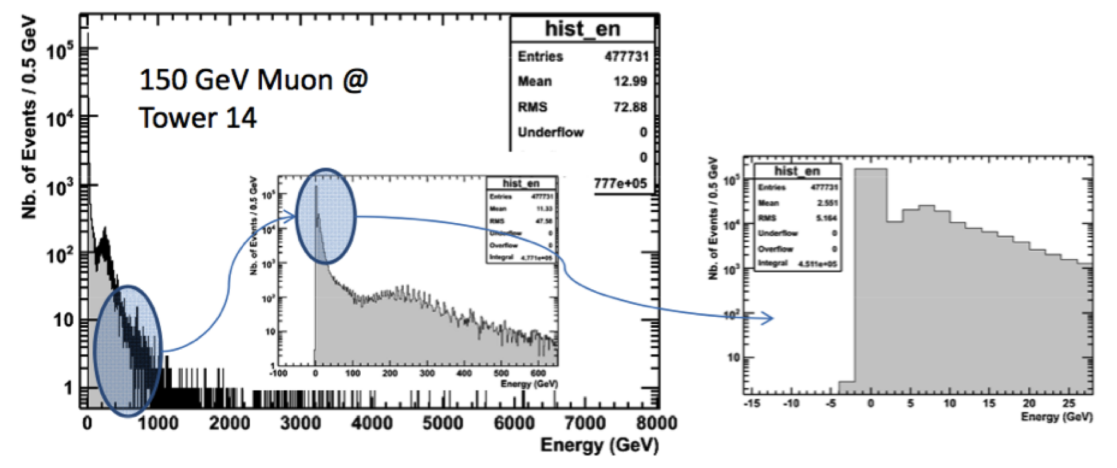

Figure 1: Muon response of HF R7525 PMTs at $150 \mathrm{GeV}$ muon test beam.

In addition to PMT replacement, the back-end electronic readout system was upgraded to Micro Telecomunications Computing Architecture ( $\mu \mathrm{TCA}$ ) readout. Also, 72 readout boxes (RBXs) with new cable design were upgraded and calibration and monitoring systems were improved [3].

\section{Characterization of Multi-Anode PMTs}

New multi-anode R7600U-200-M4 PMTs have four anodes, thinner window $(<1 \mathrm{~mm})$ and a metal envelope. Their characterisations at $800 \mathrm{~V}$ are high quantum efficiency (38 - 39\%), high gain $\left(>10^{6}\right)$, low dark current $(<1 \mathrm{nA})$ and fast timing (rise time $<2 \mathrm{~ns}$, transit time $<25 \mathrm{~ns}$ and pulse width $<15 \mathrm{~ns}$ ) [4]. They provide many improvements. Thin glass window reduces the signal size of window hit events. Multi anodes allow tagging window hit events and correct the energy of such events. Higher quantum efficiency and gain improve the resolution of the calorimeter and the dynode structure makes PMTs less susceptible to magnetic field.

The characterization tests of new PMTs were done at the University of Iowa PMT test station. All the tests were performed in light-tight boxes. Each PMT was tested for gain, dark current, and 
timing at the range of operating voltages $600-900 \mathrm{~V}$ in increments of $50 \mathrm{~V}$. Figure 2 shows the gain (left) and dark current (right) distributions of R7600U-200-M4 PMTs at 800 V. The mean (RMS) gain and dark current respectively are $2.44 \times 10^{6}\left(1.19 \times 10^{6}\right)$ and $0.665(0.770) \mathrm{nA}$.
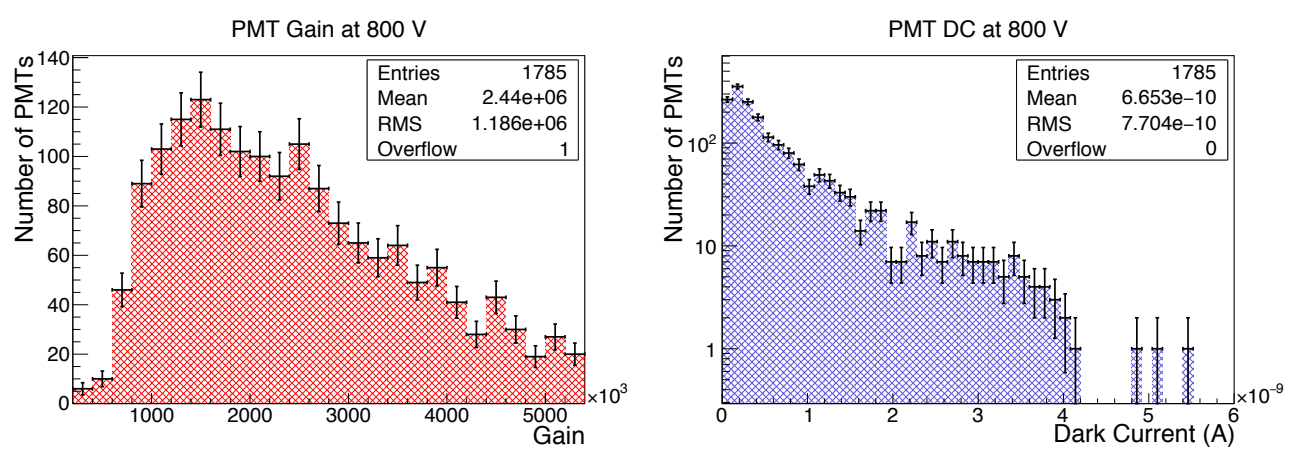

Figure 2: Gain (left) and dark current (right) distributions for R7600U-200-M4 PMTs at 800 V.

Figure 3 shows rise time (top left), transit time (top right) and pulse width (bottom) distributions of R7600U-200-M4 PMTs at $800 \mathrm{~V}$. The mean (RMS) of rise time, transit time and pulse width are respectively, $2.32(0.12) \mathrm{ns}, 5.50(0.23) \mathrm{ns}, 5.21(0.18) \mathrm{ns}$.
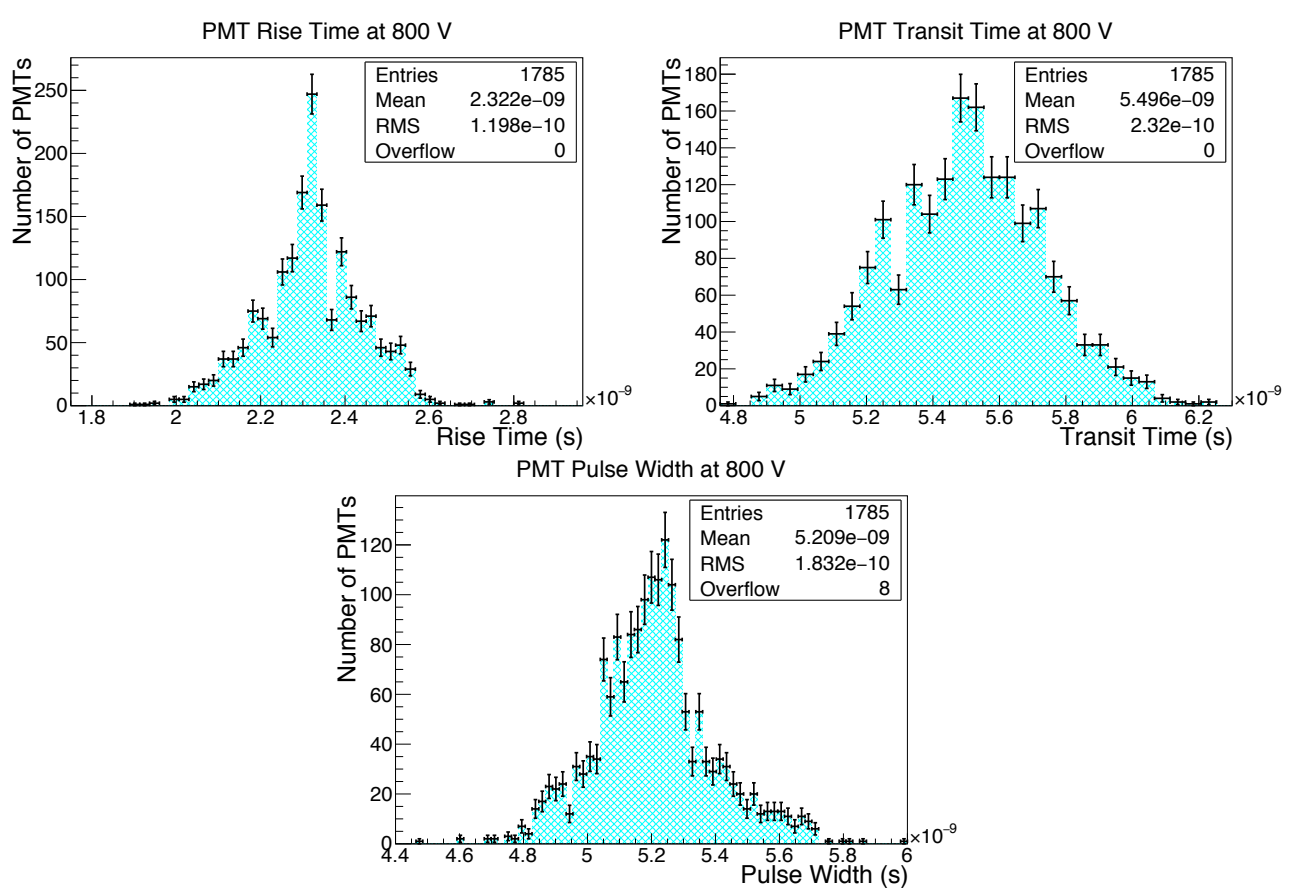

Figure 3: Rise time (top left), transit time (top right) and pulse width (bottom) distributions for R7600U200-M4 PMTs at 800 V.

\section{Front-end and Back-end Electronic Replacement}

New back-end electronics are based on $\mu$ TCA system. Figure 4 shows the crate layout structure of the $\mu$ TCA-based back-end electronics [3]. The new modules allow an increased data trans- 
fer rate; can receive data via a high-speed 5 Gbps asynchronus link and record histograms with LHC bunch crossing time resolution.

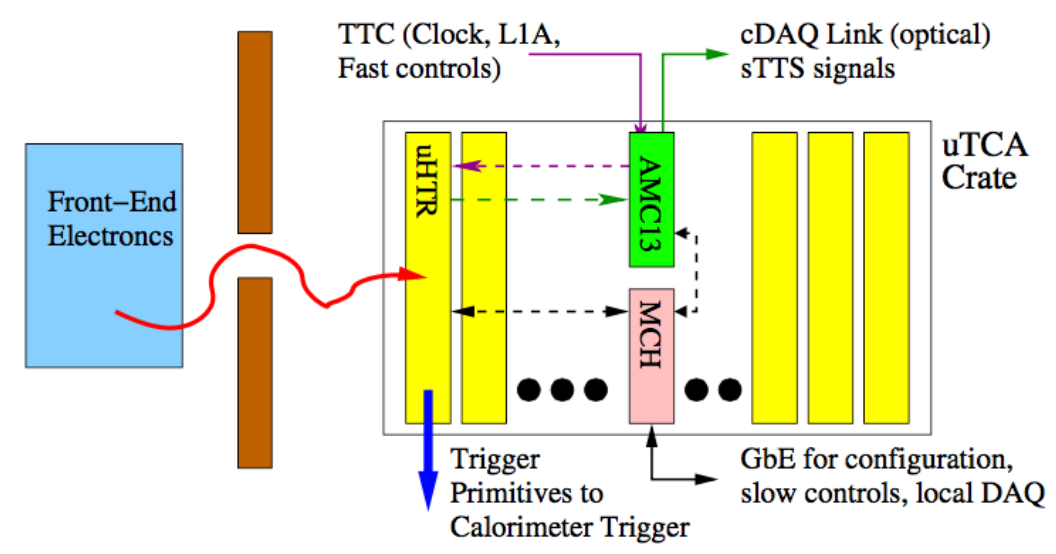

Figure 4: Crate layout structure of the $\mu$ TCA-based back-end electronics.

The front-end electronics will also be replaced during the Extended Year-End Technical Stop of 2016 - 2017. The charge integrator and encoder (QIE) will be upgraded to a new version, QIE10, which provides wider dynamic range, in between $3 \mathrm{fC}$ and $330 \mathrm{pC}$, and a dead-timeless integration and digitization of charge in 25 ns buckets.

\section{Conclusions}

The CMS Forward Hadron Calorimeters have been going through a photodetector, as well as front-end and back-end electronics upgrade. The vast majority $(\sim 99 \%)$ of new multi-anode PMTs have low dark currents, below $1 \mathrm{nA}$, and have high gain, above $10^{6}$. They show fast timing characteristics with a rise time of less than $3 \mathrm{~ns}$, transit time and pulse width less than $6 \mathrm{~ns}$. They operate stably and are now being used to collect data at the CMS experiment. These PMTs with all the improved specifications overcome large enery window event issues in the HF calorimeter, reduce fake background and make the experiment more efficient. New back-end electronics based on $\mu$ TCA provide increased data transfer. Also, upgrade front-end electronics, QIE10, will provide wider dynamic range and incorporate TDC functionality.

\section{References}

[1] CMS Collaboration, Technical proposal for the upgrade of the CMS detector through 2020, Technical Report CERN-LHCC-2011-006, CERN, Geneva, June 2011.

[2] CMS HCAL Collaboration, Study of various photomultiplier tubes with muon beams and $\check{C}$ erenkov light produced in electron showers, Journal of Instrumentation, V5-P06002, June 2010.

[3] CMS Collaboration, CMS Technical Design Report for the Phase 1 Upgrade of the Hadron Calorimeter, Technical Report CERN-LHCC-2012-015, CERN, Geneva, September, 2012.

[4] U. Akgun and et al., Characterization of 1800 Hamamatsu R7600-M4 PMTs for CMS-HF Calorimeter upgrade, Journal of Instrumentation, V9-T06005, June 2014. 